\title{
Loss of toroidal magnetic flux by emergence of bipolar magnetic regions
}

\author{
R. H. Cameron and M. Schüssler
}

\begin{abstract}
Max-Planck-Institut für Sonnensystemforschung, Justus-von-Liebig-Weg 3, 37077 Göttingen, Germany e-mail: cameron@mps.mpg.de
\end{abstract}

Received 7 December 2019 / Accepted 12 February 2020

\begin{abstract}
The polarity of the toroidal magnetic field in the solar convection zone periodically reverses in the course of the 11/22-year solar cycle. Among the various processes that contribute to the removal of "old-polarity" toroidal magnetic flux is the emergence of flux loops forming bipolar regions at the solar surface. We quantify the loss of subsurface net toroidal flux by this process. To this end, we determine the contribution of an individual emerging bipolar loop and show that it is unaffected by surface flux transport after emergence. Together with the linearity of the diffusion process this means that the total flux loss can be obtained by adding the contributions of all emerging bipolar magnetic regions. The resulting total loss rate of net toroidal flux amounts to $1.3 \times 10^{15} \mathrm{Mx} \mathrm{s}^{-1}$ during activity maxima and $6.1 \times 10^{14} \mathrm{Mx} \mathrm{s}^{-1}$ during activity minima, to which ephemeral regions contribute about 90 and $97 \%$, respectively. This rate is consistent with the observationally inferred loss rate of toroidal flux into interplanetary space and corresponds to a decay time of the subsurface toroidal flux of about 12 years, also consistent with a simple estimate based on turbulent diffusivity. Consequently, toroidal flux loss by flux emergence is a relevant contribution to the budget of net toroidal flux in the solar convection zone. The consistency between the toroidal flux loss rate due to flux emergence and what is expected from turbulent diffusion, and the similarity between the corresponding decay time and the length of the solar cycle are important constraints for understanding the solar cycle and the Sun's internal dynamics.
\end{abstract}

Key words. Sun: magnetic fields - Sun: activity

\section{Introduction}

The solar dynamo consists of poloidal magnetic field being wound up to generate toroidal magnetic field, while a process involving the Coriolis force creates poloidal field from the toroidal field (see reviews by, e.g. Ossendrijver 2003; Charbonneau 2010, 2014; Cameron et al. 2017; Brun \& Browning 2017). The oscillatory nature of the solar cycle together with Hale's polarity rules (Hathaway 2015) imply a polarity reversal of the toroidal flux system within the convection zone during each eleven-year cycle. Therefore, the question arises as to how the "old-polarity" toroidal flux is disposed of before it is replaced by the "newpolarity" flux. Principally, four different mechanism could contribute: (1) "Unwinding" by the action of differential rotation on the new (reversed) poloidal field; (2) cancellation of oppositepolarity magnetic flux at the equatorial plane due to latitudinal transport of toroidal flux by meridional flow, turbulent diffusion and/or pumping, or dynamo wave propagation (e.g. Cameron \& Schüssler 2016); (3) O-type neutral point dissipation along the dipole axis; or (4) loss through the surface due to flux emergence. The first two possibilities are discussed in Wang \& Sheeley (1991), as is the fourth possibility which they discount for the same reasons as put forward by Parker (1984) and by Vainshtein \& Rosner (1991). These latter authors pointed out that (nearly) perfect flux freezing implies a necessity to detach the magnetic field lines from their mass load in order to be able to escape from the solar interior. Flux emergence in the form of loops could provide a path to such escape through a wellorganised sequence of reconnection events between adjacent ("sea-serpent") loops. However, such a situation is considered to be rather artificial and in fact is not supported by observations. The last process has also been considered as a non-linearity limiting the amplitude of the dynamo process (e.g. Leighton 1969; Schmitt \& Schüssler 1989).

In this paper, we consider the problem of toroidal flux loss by flux emergence from a somewhat different perspective. We consider the net toroidal flux integrated over a hemispheric meridional section, $\int\left\langle B_{\phi}(r, \theta)\right\rangle \mathrm{d} S$, where $\left\langle B_{\phi}\right\rangle$ is the azimuthally averaged magnetic field (Cameron \& Schüssler 2015). For a reduction of $\left\langle B_{\phi}\right\rangle$ and thus of the net toroidal flux, toroidal field lines are not required to completely detach from the solar interior: each single emergence of a loop reduces $\left\langle B_{\phi}\right\rangle$ proportional to the width and the flux of the loop. We show that the contribution of each emerged loop to the reduction of the net toroidal flux remains constant during the subsequent evolution of the emerged flux. Therefore, the total amount of flux loss can be estimated by simply adding up the contributions of all flux-emergence events. Eventually, the corresponding amount of toroidal flux is carried away from the Sun by the solar wind and coronal mass ejections (Bieber \& Rust 1995).

The paper is organised as follows. In Sect. 2 we consider the evolution of the net toroidal flux using the procedure developed by Cameron \& Schüssler (2015). In Sect. 3 we discuss the effect of loop emergence and the subsequent surface evolution of flux on the net hemispheric toroidal flux. Quantitative estimates for the resulting loss of net toroidal flux on the basis of observed emergence rates are determined and compared with a simple estimate based on turbulent diffusion. Section 4 contains our conclusions. 


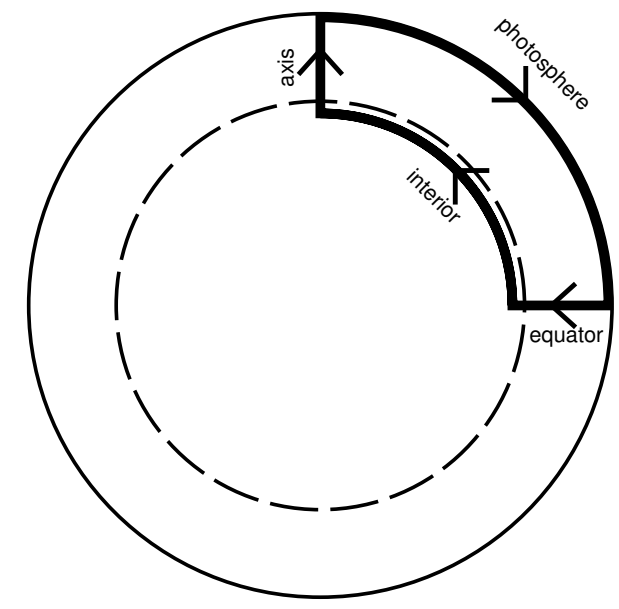

Fig. 1. Contour relevant for determining the evolution of the net toroidal flux in the northern hemisphere. The dashed line represents the base of the convection zone.

\section{Evolution of the net toroidal flux}

Hale's polarity rules and the observation that the azimuthal field at the solar surface shows a latitude-independent east-west orientation in each hemisphere during the periods of maximum activity (Cameron et al. 2018) suggest that the relevant quantity for the large-scale solar dynamo is the net hemispheric toroidal flux in the convection zone. Cameron \& Schüssler (2015) showed that the evolution equation for the net toroidal flux in the northern hemisphere (for example), $\Phi(t)$, is obtained in terms of a contour integral by integrating the hydromagnetic induction equation (neglecting the molecular diffusivity) over a hemispheric meridional section of the convection zone and applying Stokes' theorem (see Fig. 1):

$$
\frac{\mathrm{d} \Phi}{\mathrm{d} t}=\oint(\boldsymbol{U} \times \boldsymbol{B}) \cdot \mathrm{d} \boldsymbol{l}=\oint\left(\langle\boldsymbol{U}\rangle \times\langle\boldsymbol{B}\rangle+\left\langle\boldsymbol{U}^{\prime} \times \boldsymbol{B}^{\prime}\right\rangle\right) \cdot \mathrm{d} \boldsymbol{l} .
$$

Here $\boldsymbol{U}$ is the velocity field and $\boldsymbol{B}$ is the magnetic field. Quantities in angular brackets, $\langle\ldots\rangle$, are azimuthal averages and primed quantities represent fluctuations with respect to the average. This equation describes both the generation of net flux by differential rotation and the loss of flux. In particular, flux loss by transport through the surface is included in the surface part of the contour integral,

$$
\begin{aligned}
\left(\frac{\mathrm{d} \Phi}{\mathrm{d} t}\right)_{\text {surf }}= & \int_{0}^{\pi / 2}\left(\left\langle U_{\phi}\right\rangle\left\langle B_{r}\right\rangle-\left\langle U_{r}\right\rangle\left\langle B_{\phi}\right\rangle\right. \\
& \left.+\left\langle U_{\phi}^{\prime} B_{r}^{\prime}\right\rangle-\left\langle U_{r}^{\prime} B_{\phi}^{\prime}\right\rangle\right)\left.\right|_{R_{\odot}} R_{\odot} \mathrm{d} \theta
\end{aligned}
$$

in spherical polar coordinates. The first term of the integrand, $\left\langle U_{\phi}\right\rangle\left\langle B_{r}\right\rangle$, represents the effect of winding or unwinding by latitudinal differential rotation. The second term, $\left\langle U_{r}\right\rangle\left\langle B_{\phi}\right\rangle$, vanishes since there is no mean radial flow at the solar surface. The third term, $\left\langle U_{\phi}^{\prime} B_{r}^{\prime}\right\rangle$, is negligible apart during flux-emergence events. This is because the evolution of the surface flux after emergence is well represented by passive transport independent of magnetic polarity, as demonstrated by the success of surface flux transport simulations in reproducing the observations (Wang et al. 1989; Whitbread et al. 2017; Jiang et al. 2014, 2015). The fourth term, $\left\langle U_{r}^{\prime} B_{\phi}^{\prime}\right\rangle$, represents flux emergence and submergence. The latter process takes place when magnetic features of opposite polarities meet and cancel after reconnection.

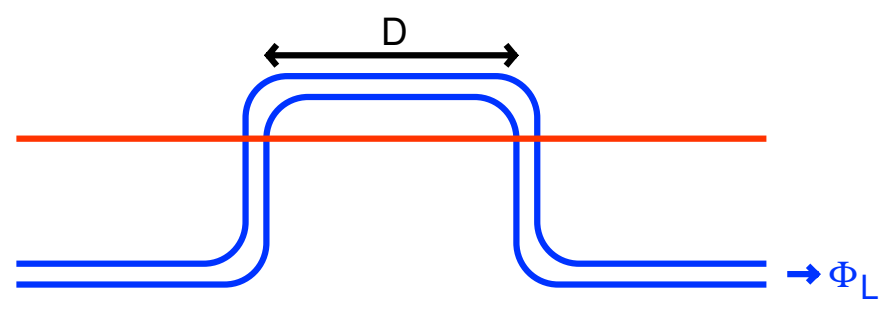

Fig. 2. Idealised sketch of an emerged loop of toroidal magnetic flux. The flux tube (in blue) has a magnetic flux of $\Phi_{\mathrm{L}}$; the longitudinal extent of the emerged part is $D$. The photosphere is indicated by the red line.

\section{Flux loss by flux emergence}

As we see above, flux emergence and submergence change the net toroidal magnetic flux in the convection zone. The evolution of bipolar regions after emergence is well described as passive flux transport by horizontal flows (differential rotation, meridional circulation, and convective flows described in terms of turbulent diffusion). Submergence is represented by diffusive flux cancellation at locations where opposite polarities meet and one might therefore expect the amount of toroidal flux loss to change in the course of the evolution of a bipolar region. However, we show in this section that this is not the case and furthermore that the total amount of flux loss can be quantitatively estimated by simply adding the contributions of all bipolar magnetic regions.

Considering a single emerged loop of toroidal magnetic flux as sketched in Fig. 2, the resulting decrease of the azimuthal average, $\left\langle B_{\phi}\right\rangle$, corresponds to a reduction, $\Delta \Phi_{\text {tor }}$, of the subsurface net toroidal flux, given by

$\Delta \Phi_{\text {tor }}=\frac{D \Phi_{\mathrm{L}}}{2 \pi R_{\odot} \cos \lambda}$

where $\Phi_{\mathrm{L}}$ is the amount of flux contained in the loop, $D$ is the longitudinal extension of the loop after emergence, and $\lambda$ its position in latitude. Equation (3) follows from the fact that the azimuthally averaged change in the subsurface toroidal flux due to the emergence is equal to the flux of the loop multiplied by the fraction of the longitudinal separation of the two polarities at the surface to the circumference of the Sun at the latitude of emergence. More simply, the change in the longitudinally averaged subsurface toroidal flux due to an emergence is the flux of the emerging flux tube multiplied by the fraction of the tube in longitude which has moved across the photosphere. Various processes can in principle affect the subsequent evolution of the emerged flux contained in the corresponding bipolar magnetic region (BMR): (1) transport by horizontal convective flows, which can be described as turbulent diffusion (random walk; see Leighton 1964); (2) latitudinal differential rotation acting on tilted BMRs; (3) meridional flow; and (4) longitudinal drift of the two polarities in opposite directions caused by magnetic tension in the subsurface part of the loop (van Ballegooijen 1982). Surface flux transport simulations have repeatedly demonstrated that the surface magnetic flux is passively transported by the surface flows, and therefore the fourth (dynamic) process seems irrelevant for the evolution of the net toroidal magnetic flux. While Eq. (2) implies that meridional flow does not affect the net toroidal magnetic flux, latitudinal differential rotation leads to the "unwinding" and eventual reversal of the net toroidal flux in the course of the dynamo process.

The question remains as to how far the first process, horizontal turbulent diffusion, which causes cancellation, dispersal, and reconnection of the emerged surface flux, leads to a temporal change of the amount of flux loss given by Eq. (3). The 
relevant properties of the process in this regard are that (1) diffusion is symmetric (independent of polarity), that is, it affects both polarities of the loop flux in the same way, and (2) diffusion is a linear process, meaning that the effects of many BMRs can be simply determined by adding together the contributions of the individual BMRs, thus automatically taking into account the permanent reorganisation of the surface field by reconnection and cancellation of magnetic flux. It therefore suffices to solely consider the evolution of one loop.

In the course of the diffusive evolution, both oppositepolarity patches of the vertical loop flux spread in all horizontal directions. While Eq. (2) shows that expansion in latitude does not affect the subsurface net toroidal flux, spreading in the longitudinal direction potentially could. Part of the emerged flux cancels at the neutral line between the polarities and thus "heals" the subsurface toroidal flux. Another part of the flux expands longitudinally away from the neutral line and eventually diffuses all around the Sun, thus finally removing the corresponding amount of toroidal flux. While the cancellation at the neutral line reduces the amount of flux loss, the expanding part increases the flux loss by effectively enlarging the polarity separation, $D$. Owing to flux conservation and the symmetry of the diffusion process, it turns out that both contributions exactly balance each other, meaning that the loss of net toroidal flux, $\Delta \Phi_{\text {tor }}$, remains time-independent at its initial value given by Eq. (3). This can be seen formally by the following illustrative calculation.

Assume, for simplicity, one-dimensional cartesian geometry with a purely vertical field, $B(x, t)$, that depends on the horizontal coordinate, $x$ (representing the longitudinal direction), and time, $t$, in an infinite domain. Consider the evolution by diffusion of a bipolar region of vertical flux that is centred at $x=0$ with the two polarities centred at $x= \pm x_{0}$. The evolution of both polarities can be described by the analytical solution for the diffusive spread of an initial delta function in terms of Gaussian profiles, that is,

$B(x, t)=B_{0} \sqrt{\frac{a}{\pi}}\left[e^{-a\left(x-x_{0}\right)^{2}}-e^{-a\left(x+x_{0}\right)^{2}}\right]$,

with $a=(4 \eta t)^{-1}$ and diffusivity $\eta$. The centre of gravity of the field distribution for $x \geq 0$ is given by

$\bar{x}_{+}=\frac{\int_{0}^{\infty} B x \mathrm{~d} x}{\int_{0}^{\infty} B \mathrm{~d} x}$

The centre of gravity, $\bar{x}_{-}$for $x \leq 0$ is defined analogously. The symmetry of the configuration entails $\bar{x}_{-}=-\bar{x}_{+}$. The relevant quantity for the reduction of the subsurface horizontal flux, corresponding to $D \Phi_{\mathrm{L}}$ in Eq. (3), is given by

$R(t)=\bar{x}_{+} \int_{0}^{\infty} B \mathrm{~d} x+\bar{x}_{-} \int_{-\infty}^{0} B \mathrm{~d} x=2 \int_{0}^{\infty} B x \mathrm{~d} x$,

again owing to symmetry. Using Eq. (4) we obtain

$$
\int_{0}^{\infty} B x \mathrm{~d} x=B_{0} \sqrt{\frac{a}{\pi}}\left(I_{+}-I_{-}\right),
$$

with

$$
\begin{aligned}
& I_{+}=\int_{0}^{\infty} x e^{-a\left(x-x_{0}\right)^{2}} \mathrm{~d} x \\
& I_{-}=\int_{0}^{\infty} x e^{-a\left(x+x_{0}\right)^{2}} \mathrm{~d} x .
\end{aligned}
$$

After some elementary algebra we obtain

$\left(I_{+}-I_{-}\right)=2 x_{0} \int_{x_{0}}^{\infty} e^{-a\left(x-x_{0}\right)^{2}} \mathrm{~d} x=x_{0} \sqrt{\frac{\pi}{a}}$,

meaning that with Eq. (6) we have

$R(t)=2 B_{0} \sqrt{\frac{a}{\pi}} \cdot x_{0} \sqrt{\frac{\pi}{a}}=2 B_{0} x_{0}$,

which is independent of time. This means that the diffusive evolution of a bipolar magnetic region does not change the reduction of the net toroidal flux due to its emergence, which is given by Eq. (3). The increase of flux loss by the outward spreading of the magnetic flux at the surface is exactly balanced by flux cancellation. In fact, this result does not depend on the special assumption of Gaussian profiles but is valid for any symmetric profile that is uniformly stretched while keeping the integral constant.

Since the flux loss, $\Delta \Phi_{\text {tor }}$, associated with an individual bipolar region is time-independent and diffusion is a linear process, we can estimate the mean rate of flux loss during a time interval $\Delta t$ by simply adding the individual contributions given by Eq. (3) of the bipolar regions emerging within that time, that is,

$\frac{\mathrm{d} \Phi_{\text {tor }}}{\mathrm{d} t}=\frac{\gamma \sum_{i}\left(D \Phi_{\mathrm{L}}(\cos \lambda)^{-1}\right)_{i}}{2 \pi R_{\odot} \Delta t}$.

The factor $\gamma$ is the fraction of the emerged flux that is not balanced by emergences with the opposite polarity orientation, i.e. $\gamma=\left(\Phi_{\text {Hale }}-\Phi_{\text {non-Hale }}\right) /\left(\Phi_{\text {Hale }}+\Phi_{\text {non-Hale }}\right)$, where $\Phi_{\text {Hale }}$ and $\Phi_{\text {non-Hale }}$, respectively, are the amounts of flux that emerge obeying Hale's law and not obeying it.

We first consider the contribution due to ephemeral regions, small bipolar regions carrying a magnetic flux of the order of $10^{20} \mathrm{Mx}$ that emerge ubiquitously at the solar surface. Hagenaar (2001) determined a value of $5 \times 10^{23} \mathrm{Mx}$ per day for the emergence rate of unsigned flux in ephemeral regions over the entire solar surface. About $60 \%$ of these were found to obey Hale's polarity laws (i.e. a surplus of 20\%), and therefore $\gamma=0.2$ in this case. For a rough estimate of the corresponding loss of toroidal flux we assume that polarity separation, $D$, loop flux, $\Phi_{\mathrm{L}}$, and emergence latitude are all uncorrelated. Since the contribution of each emerging loop to the total unsigned surface flux is equal to $2 \Phi_{\mathrm{L}}$, we have $\sum_{i} \Phi_{\mathrm{L}}=2.5 \times 10^{23} \mathrm{Mx}$ per day. The average polarity separation for ephemeral regions is about $9 \mathrm{Mm}$ (Hagenaar 2001). Since $D$ is the longitudinal separation, we have $D_{i}=9 \cos \left(\alpha_{i}\right)$ Mm where $\alpha_{i}$ is the tilt angle of the axis of the ephemeral region with respect to the east-west direction. For a given longitudinal polarity orientation (Hale or anti-Hale), these angles are likely to be uniformly distributed between $\pm 90^{\circ}$, meaning that on average we expect $\left\langle D_{i}\right\rangle \approx 9 \times 0.64=5.76 \mathrm{Mm}$ where 0.64 is the average value of $\cos (\alpha)$ between $-90^{\circ}$ and $90^{\circ}$. We assume the emergences occur uniformly over the surface, and therefore the weighted average of $\cos (\lambda)^{-1}$ over the emergences is

$$
\begin{aligned}
\left\langle(\cos \lambda)^{-1}\right\rangle_{i} & =\frac{\int_{0}^{90^{\circ}} \cos (\lambda)^{-1} \cos \lambda \mathrm{d} \lambda}{\int_{0}^{90^{\circ}} \cos \lambda \mathrm{d} \lambda} \\
& =\pi / 2,
\end{aligned}
$$

(8) where the weighting factor $\cos \lambda$ accounts for the fact that the length of the circumference at constant latitude is proportional to 
$\cos \lambda$. We thus obtain for the loss rate of toroidal flux per hemisphere due to the emergence of ephemeral regions a value of

$\frac{\mathrm{d} \Phi_{\text {tor,hem }}^{\mathrm{ER}}}{\mathrm{d} t} \approx 5.9 \times 10^{14} \mathrm{Mx} \mathrm{s}^{-1}$.

The results of Hagenaar (2001) are based on data from October 1997 , that is, under solar minimum conditions. Since the emergence rate of ephemeral regions varies by a factor of approximately two or three during the solar cycle (Harvey et al. 1975; Martin \& Harvey 1979; Hagenaar et al. 2003), we expect the loss rate during solar maxima to be correspondingly higher, so that

$\left.\frac{\mathrm{d} \Phi_{\text {tor,hem }}^{\mathrm{ER}}}{\mathrm{d} t}\right|_{\text {maximum }} \approx 2 \times 5.9 \times 10^{14} \mathrm{Mx} \mathrm{s}^{-1}$.

We note that a few years before activity minima ephemeral regions from the current and the next cycle are both present on the surface, and the change in the net hemispheric subsurface toroidal flux will reflect the difference between the contributions of the old and new cycle ephemeral regions.

For active regions exceeding 3.5 square degrees in size, Schrijver \& Harvey (1994) report emergence rates over the entire solar surface of $7.4 \times 10^{20} \mathrm{Mx}$ per day during activity minimum and $6.2 \times 10^{21} \mathrm{Mx}$ per day during maximum. For simplicity, we assume an average polarity separation of $40 \mathrm{Mm}$, east-west alignment, and emergence close to the equator $\cos \lambda=1$. We then obtain

$\frac{\mathrm{d} \Phi_{\text {tor,hem }}^{\mathrm{AR}}}{\mathrm{d} t} \approx 2.0 \times 10^{13} \mathrm{Mx} \mathrm{s}^{-1}$,

during activity minimum and

$\frac{\mathrm{d} \Phi_{\text {tor,hem }}^{\mathrm{AR}}}{\mathrm{d} t} \approx 1.6 \times 10^{14} \mathrm{Mx} \mathrm{s}^{-1}$,

during maximum. Assuming a factor of two variation of the emergence rate of ephemeral regions between minimum and maximum, these regions therefore contribute about $90 \%$ of the total loss rate of toroidal flux during solar maxima and about 97\% during minima. The total flux loss rate per hemisphere during minimum is therefore

$\left.\left(\frac{\mathrm{d} \Phi_{\text {tor,hem }}^{\mathrm{ER}}}{\mathrm{d} t}+\frac{\mathrm{d} \Phi_{\text {tor,hem }}^{\mathrm{AR}}}{\mathrm{d} t}\right)\right|_{\text {minimum }}=6.1 \times 10^{14} \mathrm{Mx} \mathrm{s}^{-1}$,

and $1.3 \times 10^{15} \mathrm{Mx} \mathrm{s}^{-1}$ during maximum. With the total loss rate of $1.3 \times 10^{15} \mathrm{Mx} \mathrm{s}^{-1}$ around maxima and a total amount of subsurface toroidal flux of $5 \times 10^{23} \mathrm{Mx}$ per hemisphere (Cameron \& Schüssler 2015), we obtain a characteristic decay time of 12.2 years. Consequently, flux loss through the photosphere associated with flux emergence is an important factor for the evolution of the subsurface toroidal flux on solar-cycle timescales. Roughly approximating the cycle-averaged loss rate based on the mean of its maximum and minimum values, i.e., $9.6 \times 10^{14} \mathrm{Mx} \mathrm{s}^{-1}$, we obtain a total loss of toroidal flux by flux emergence over 11 years of $3.3 \times 10^{23} \mathrm{Mx}$. Bieber \& Rust (1995) estimated the total loss of toroidal flux into interplanetary space as $10^{24} \mathrm{Mx}$ per eleven-year cycle, that is, $5 \times 10^{23} \mathrm{Mx}$ per hemisphere per cycle. This is roughly consistent with our result of $3.3 \times 10^{23} \mathrm{Mx}$ per hemisphere per cycle, given the considerable uncertainties and simplifications entering both estimates.

The rate at which BMRs appear on the solar surface as a function of the amount of flux that emerges is described by a single power law which extends over five orders of magnitude from small ephemeral regions to large active regions (see, e.g. Hagenaar et al. 2003; Thornton \& Parnell 2011). Unlike active regions which emerge only at latitudes of less than about $40^{\circ}$, ephemeral regions emerge all over the solar surface. However ephemeral regions emerging in the butterfly wings have a tendency to obey Hale's law, with the same east-west orientation as the active regions of the same cycle (Martin \& Harvey 1979). The tendency of an ephemeral region to emerge obeying Hale's law extends the butterfly wings to earlier times and higher latitudes (Martin \& Harvey 1979; Wilson et al. 1988).

Which size ranges of BMRs are most important for the loss of toroidal field through the surface is mainly decided by the competition between the number of emergences and their tendency to obey Hale's law. The ephemeral regions dominate the flux loss at all phases of the solar cycle because ephemeral region emergence is much more common than active region emergence. The larger ephemeral regions in the range of $10^{18} \mathrm{Mx}$ and above are presumably more important than the smaller emergences because the tendency to obey Hale's law decreases rapidly with decreasing flux of the BMR (Hagenaar et al. 2003).

We can also compare our result with simple estimates in terms of turbulent diffusion. Instead of regarding individual emergence events, this approach considers the transport of toroidal magnetic field by turbulent motions throughout the convection zone and across the photosphere. Ignoring turbulent pumping, one can parameterize this by an effective turbulent diffusivity, $\eta_{\mathrm{t}}$, the value of which can be estimated using mixing length theory (e.g. Muñoz-Jaramillo et al. 2011), from numerical simulations (e.g. Warnecke et al. 2018), or can be inferred from observations (e.g. Cameron \& Schüssler 2016). Near-surface values of $\eta_{\mathrm{t}}$ are typically around $10^{12} \mathrm{~cm}^{2} \mathrm{~s}^{-1}$. Using the depth of the convection zone, $L=200 \mathrm{Mm}$, as a typical length scale, this leads to a diffusive decay time of $\tau=L^{2} / \eta_{\mathrm{t}} \simeq 12.7$ years, which is consistent with the above value of 12.2 years from flux emergence.

\section{Conclusions}

Our results show that the loss of net toroidal flux from the solar interior due to flux emergence can be faithfully estimated by adding the time-independent contributions of the individual bipolar regions to the reduction of the longitudinally averaged azimuthal field. Using the observed emergence rates of ephemeral and active regions leads to a characteristic decay time of the toroidal flux of about 12 years, in which ephemeral regions contribute most of the effect. The decay rate of toroidal flux by flux emergence is also consistent with simple estimates based on turbulent diffusion. Consequently, flux emergence represents a relevant loss mechanism for the interior toroidal flux. The decay of toroidal flux is further enhanced by cancellation across the equator, dissipation along the dipole axis, and "unwinding" by differential rotation. However, these processes are presumably not dominant because the toroidal flux loss through the photosphere already accounts for most of what needs to be removed. The twelve-year timescale for toroidal flux loss due to flux emergence is close to the eleven-year solar cycle period. This means that the flux loss is very important to the subsurface flux evolution, and the twelve-year timescale is an important constraint for models of the solar dynamo.

Acknowledgements. RHC acknowledges partial support from ERC Synergy grant WHOLE SUN 810218. 
R. H. Cameron and M. Schüssler: Loss of toroidal magnetic flux

\section{References}

Bieber, J. W., \& Rust, D. M. 1995, ApJ, 453, 911

Brun, A. S., \& Browning, M. K. 2017, Liv. Rev. Sol. Phys., 14, 4

Cameron, R., \& Schüssler, M. 2015, Science, 347, 1333

Cameron, R. H., \& Schüssler, M. 2016, A\&A, 591, A46

Cameron, R. H., Dikpati, M., \& Brandenburg, A. 2017, Space Sci. Rev., 210, 367

Cameron, R. H., Duvall, T. L., Schüssler, M., \& Schunker, H. 2018, A\&A, 609, A56

Charbonneau, P. 2010, Liv. Rev. Sol. Phys., 7, 3

Charbonneau, P. 2014, ARA\&A, 52, 251

Hagenaar, H. J. 2001, ApJ, 555, 448

Hagenaar, H. J., Schrijver, C. J., \& Title, A. M. 2003, ApJ, 584, 1107

Harvey, K. L., Harvey, J. W., \& Martin, S. F. 1975, Sol. Phys., 40, 87

Hathaway, D. H. 2015, Liv. Rev. Sol. Phys., 12, 12

Jiang, J., Hathaway, D. H., Cameron, R. H., et al. 2014, Space Sci. Rev., 186, 491

Jiang, J., Cameron, R. H., \& Schüssler, M. 2015, ApJ, 808, L28
Leighton, R. B. 1964, ApJ, 140, 1547

Leighton, R. B. 1969, ApJ, 156, 1

Martin, S. F., \& Harvey, K. H. 1979, Sol. Phys., 64, 93

Muñoz-Jaramillo, A., Nandy, D., \& Martens, P. C. H. 2011, ApJ, 727, L23

Ossendrijver, M. 2003, A\&ARv, 11, 287

Parker, E. N. 1984, ApJ, 281, 839

Schmitt, D., \& Schüssler, M. 1989, A\&A, 223, 343

Schrijver, C. J., \& Harvey, K. L. 1994, Sol. Phys., 150, 1

Thornton, L. M., \& Parnell, C. E. 2011, Sol. Phys., 269, 13

Vainshtein, S. I., \& Rosner, R. 1991, ApJ, 376, 199

van Ballegooijen, A. A. 1982, A\&A, 106, 43

Wang, Y. M., \& Sheeley, Jr., N. R. 1991, ApJ, 375, 761

Wang, Y. M., Nash, A. G., \& Sheeley, Jr., N. R. 1989, ApJ, 347, 529

Warnecke, J., Rheinhardt, M., Tuomisto, S., et al. 2018, A\&A, 609, A51

Whitbread, T., Yeates, A. R., Muñoz-Jaramillo, A., \& Petrie, G. J. D. 2017, A\&A, 607, A76

Wilson, P. R., Altrocki, R. C., Harvey, K. L., Martin, S. F., \& Snodgrass, H. B. 1988, Nature, 333, 748 